\title{
Of targets and anti-targets
}

The success of some targeted anticancer drugs, in particular kinase inhibitors, is known to be due to a certain amount of off-target activity (polypharmacology). Since kinase signalling cascades are complex and interconnected, it is thought that simultaneous inhibition of several signalling 'nodes' is needed to prevent the rapid emergence of resistance to targeted agents. However, to date, the design of anticancer agents with favourable polypharmacology has been guided by serendipity rather than intent. Now, reporting in Nature, the groups of Shokat and Cagan present a systems pharmacology approach that allows the identification of targets and anti-targets for the rational design of drugs with an optimized polypharmacological profile.

Combining aspects of target and phenotype-based drug discovery, the authors used a Drosophila melanogaster model of multiple endocrine neoplasia type 2 (MEN2), kinase profiling and genetics in a stepwise approach. The MEN2 model is based on an oncogenic RET transgene $\left(\mathrm{RET}^{\mathrm{MEN} 2}\right.$ ) targeted to developing epithelia such as wing, eye and leg using the patched promotor, with the transgene dose calibrated to permit $50 \%$ survival of D. melanogaster larvae to pupariation and $0 \%$ survival to adulthood. Oral administration of clinical kinase inhibitors such as vandetanib, sunitinib or sorafenib resulted in different levels of suppression of RET-driven lethality - sorafenib was most potent with rescue of about $17 \%$ of animals to adulthood. The authors then screened a library of compounds that target RET in addition to other classes of kinases, and they identified one compound, $\mathrm{AD} 57$, that rescued $25 \%$ of the animals to adulthood. It suppressed defects in wing and eye development and had an improved efficacy and toxicity profile compared to clinical kinase inhibitors.

In vitro experiments showed that AD57 also inhibited the viability of patientderived cell lines with RET $^{\mathrm{MEN} 2}$ mutations. In a mouse xenograft model of RET-driven tumours, AD57 potently suppressed tumour growth and showed no toxicity, validating the $D$. melanogaster model as a useful tool for the discovery of compounds with improved efficacy and toxicity profiles.

Using kinase profiling and further genetic and pharmacological manipulation in D. melanogaster, AD57 and its derivatives were analysed with regards to their kinase inhibition profiles and the relative contributions of their targets to the efficacy and toxicity of the compound. Small alterations of the structure of AD57 were shown to lead to considerable changes in kinase selectivity. For example, AD58, a close analogue of AD57, induced whole-animal toxicity, providing an opportunity to explore aspects of $\mathrm{AD}$ class drug toxicity. It was found that ERK and SRC inhibition suppressed oncogenic RET signalling, whereas increased RAS pathway signalling contributed to toxicity. AD58 was found to exert its toxic effects through the inhibition of target of rapamycin (TOR), which was thought to be due to the fact that TOR signalling provides feedback inhibition of the RAS pathway.

These insights were used to generate close $\mathrm{AD} 57$ analogues with optimized polypharmacological profiles. AD80 and AD81, in which TOR binding was abolished, were shown to rescue 70-90\% of MEN2 Drosophila larvae, and AD80 had an improved efficacy and toxicity profile in mammalian MEN2 models compared to AD57 and vandetanib.

The authors caution that the true predictive value of this approach awaits further testing. However, they point out that the development of drugs with optimized polypharmacology has distinct advantages over the related approach of using drug combinations, as the latter is hampered by the cost and complexity of clinical trials, complex targetprofile interactions and differing pharmacokinetics.

Alexandra Flemming

ORIGINAL RESEARCH PAPER Dar, A. C. et al.

Chemical genetic discovery of targets and anti-targets for cancer polypharmacology. Nature 486, 80-84 (2012) 